\title{
Optimization of THz Absorption in Thin Films
}

\author{
Dragoslav Grbovic, Fabio Alves, Brian Kearney, Karamitros Apostolos, Gamani Karunasiri \\ Physics Department \\ Naval Postgraduate School \\ Monterey, CA 93943 \\ dgrbovic@nps.edu
}

\begin{abstract}
In this paper, we report on experimentally verified models, which assist in the design and fabrication of either thin metal films, with high broad-band absorption in the 3-5 $\mathrm{THz}$ spectral region, or thin metatamaterial structures of nearly $100 \%$ absorption at a specific, resonant, THz frequency. The THz absorption properties of the structures were modeled using COMSOL finite element modeling software. Optimized thin metal films and thin metamaterial structures were fabricated using standard microfabrication processes on Si substrates. The THz spectral characteristics of the structures were probed using FTIR spectrometer in the reflection mode. The measured $\mathrm{THz}$ reflection, from thin film of both broad-band and resonant metamaterial structures, exhibit excellent agreement with their respective models. Both types of absorbers can be incorporated in the fabrication of MEMS-based $\mathrm{THz}$ thermal sensors operating in active mode with quantum cascade laser (QCL) sources.
\end{abstract}

\section{INTRODUCTION}

$\mathrm{THz}$ imaging has gained increased attention in recent years. The main motivation is the property of $\mathrm{THz}$ radiation to penetrate through most non-metalic materials and fabrics, and is therefore attractive for medical and security applications. In addition, the radiation is non-ionizing and therefore does not pose a risk for human health.

The challenge with this type of radiation is the lack of appropriate detectors as well as sufficiently powerful sources as the applications require external illumination. Modified, thermal IR imagers have successfully been used when fitted with the THz optics. However, these imaging systems are built with materials optimized for infrared (IR) radiation. Additional challenge, when trying to create sensitive IR detectors based on the MEMS bolometric or micromechanical detectors (both of which base their operation on absorbing the radiation and increasing the detector temperature) is that the dimensions that are required in those systems, such as thicknesses of absorbent films, are significantly smaller than the wavelength of radiation being detected. Thus, it is important to explore potential new approaches for achieving high $\mathrm{THz}$ absorption while keeping thin film architecture.

In terms of spectral characteristics of the thin films both broad band and narrow band absorption characteristics are desired depending on the application. For example, spectroscopic applications require sensitivity to broader range of $\mathrm{THz}$ frequencies In addition, quantum cascade lasers are emerging as the most efficient and compact sources of $\mathrm{THz}$ radiation $[1,2]$ for imaging applications, sensors highly tuned for the absorption of laser-specific frequencies would be appropriate.

\section{RELADED WORK AND BACKGROUND}

Our group has demonstrated the use of bolometer-based thermal IR cameras, fitted with $\mathrm{THz}$ optics for real-time imaging of concealed objects [3]. The goal in our previous and current work is to identify absorbing layers to embed into the existing micromechanical IR detectors [4] whose sensitivity and microfabrication simplicity makes them good candidates for $\mathrm{THz}$ imaging. This is because $\mathrm{THz}$ imaging requires much higher sensitivity compared to the IR imaging sensors as the power being detected is significantly smaller then that of the IR radiation.

In order to keep the microfabrication processes simple and with as few steps as possible, the films were limited to the standard Si-friendly materials and processes. Essentially, the goal was to replace the IR absorbing layer in the MEMS IR thermal detectors with the THz-optimized thin film or multilayered metamaterial.

\section{MODEL}

The $\mathrm{THz}$ absorption properties of the absorbers were modeled using COMSOL finite element software with two different models. The first model represented the broad-band absorber as a thin-film on a supporting wafer. The second model represented a highly-tuned metamaterial absorber as a multi-layered film. The accuracy of both models has been investigated by comparing them with experimental measurements using an FTIR spectrometer.

Both simulations and the experiments quantify the transmission and reflection and predict the absorption based on the following equation

$$
\mathrm{A}=1-\mathrm{T}-\mathrm{R},
$$

where $\mathrm{T}$ and $\mathrm{R}$ are the fractional transmission and reflection, respectively. 


\section{A. Broad-band Absorber}

Analysis of the properties of conductive thin films as absorbers has been chosen as they represent a lossy media (i.e. have a complex refractive index which is associated with absorption). In addition, the same absorptive layer can also be employed for optically probing the deflection associated with micromechanical thermal detection $[4,5]$.

In order to model the behavior of metal films, the twodimensional (2D) environment of the COMSOL multiphysics and its RF module has been utilized. As our investigation initially focused on fully-covered 4-inch Si wafers with metal films, it was possible to use the periodic boundary condition and therefore simulate the "infinite" plane of the thin film, while keeping the problem space relatively small. Since the experimental measurements had to be performed with films on the $\mathrm{Si}$ wafers, the $\mathrm{Si}$ substrate has been included into the model.

The schematics of the model is shown in Figure 1. The air above the substrate had to be split into two layers, as the finite element modeling software provides the superposition of the incoming and reflected waves in the region immediately above the substrate and film. In order to separate the reflected portion of the wave, the wave is "emitted" only in the second region from the top and directed toward the structure being analyzed. In this configuration the top region contains only the reflected portion of the wave. The region of air underneath the substrate contains the transmittied portion of the wave. The total power of the wave directed to the film and the substrate is one of the parameters of the model. Not shown in the illustration are the two "perfectly matched layer" regions on top and bottom of the model. They used in the model to represent the "infinite space".

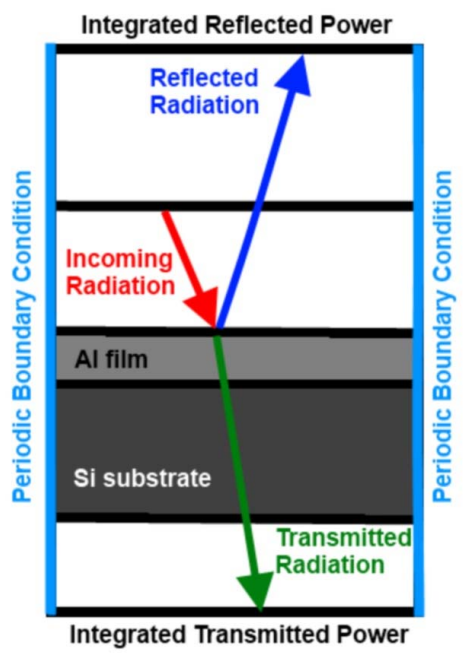

Figure 1 Schematic of the model of the film together with the substrate (substrate shown not to scale).

The RF module of COMSOL multiphysics has been used whose calculations were based on solving the Maxwell's equations. The complex refractive index of the metal films are obtained for each frequency value in our simulation, using the following equation:

$$
n_{c r}^{*}=(1-i) \sqrt{\frac{\sigma}{4 \pi \varepsilon_{0} f}}
$$

where the $\sigma$ is measured conductivity and $f$ is the frequency of the incoming radiation. Complex refractive index for $\mathrm{Si}$ substrate has been calculated as described in [6].

The reflected (R) and transmitted (T) power are obtained by integrating the power on the top and botom boundaries, respectivelly (Fig. 1), for each individual frequency of incoming radiation.

\section{B. Highly-tuned Absorber}

The second type of absorbing films investigated were periodic structures or metamaterials. The metamaterials are designed to have almost total (100\%) absorption at a frequency matched to that of a quantum cascade laser (QCL) used as the illumination source. The metamaterial film analyzed consisted of a $100 \mathrm{~nm}$ thick $\mathrm{Al}$ ground plane, 1.4 $\mu \mathrm{m}$ thick dielectric $\left(\mathrm{SiO}_{2}\right)$ layer and a layer consisting of a periodic array of $16.5 \mu \mathrm{m} \times 16.5 \mu \mathrm{m} \mathrm{Al}$ (100 nm thick) squares separated by $2 \mu \mathrm{m}$ gaps on top. Figure 2 a) illustrates one unit cell of the configuration as represented in the 3D model space. Even though these structures were also fabricated on supporting 4-inch $\mathrm{Si}$ wafers, the substrate did not need to be included in the model because the $100 \mathrm{~nm}$ of $\mathrm{Al}$ in the ground plane was sufficiently thick to prevent any transmission. This has also been verified experimentally. The experimental absorption was therefore simply $\mathrm{A}=1-\mathrm{R}$, where $\mathrm{R}$ is the fractional reflection.

Modeling a single unit cell was sufficient to estimate the total absorption. This was possible because the structure exhibits symmetry under normal incidence, as depicted in Figure 2 a). Perfect electric and perfect magnetic conductors are selected as boundary conditions on the opposite walls perpendicular to the electric and magnetic fields, respectively. This configuration represents an infinite array of unit cells shown which closely corresponds to the realistic situation where 4-inch wafers were used completely coved by these arrays. Various additional information can be extracted from the final element model. Figure 2 b) illustrates the visualization of electric field in the metal square in the top layer.

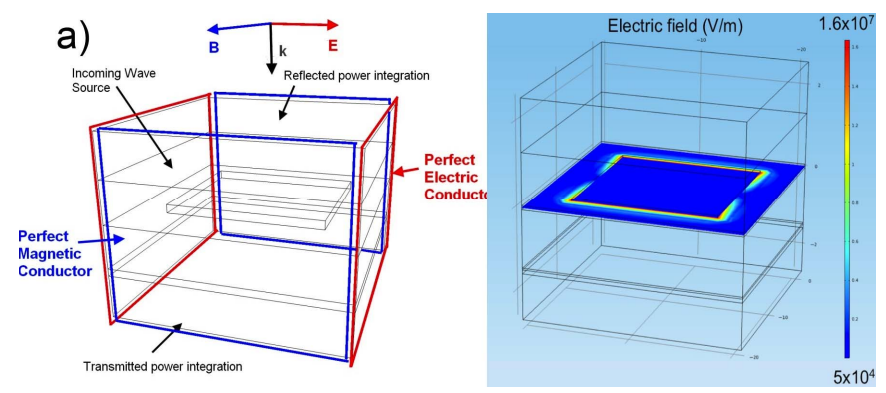

Figure 2. a) Schematic of the 3D finite element model of metamaterial unit cell; perfect electric and magnetic conductor boundary conditions are used to represent infinite array of unit cells. b) illustration of visualization of electric field as one of examples of information that can be extracted from the finite element model. 
Film parameters such as thickness and electric conductivity used in both models have been obtained from experimental measurements.

\section{SAMPLE PREPARATION}

Samples with thin metal films for broad-band absorbers have been fabricated using the e-beam evaporation. A thin film of $10 \mathrm{~nm}$ of $\mathrm{Ni}$ as been deposited on a 4-inch Si wafers. The thickness was then verified using a surface profilometer and the conductivity measurements were obtained using a 4point contact probe.

Resonant absorber samples have been fabricated using the process illustrated in the Figure 3. The ground plane of 100 $\mathrm{nm}$ of $\mathrm{Al}$ covering the wafer is initially deposited using the ebeam evaporation. The thickness is measured using the surface profilometer and conductivity is measured using the 4point probe. This step is followed by deposition of a dielectric film of $1.4 \mu \mathrm{m}$ of $\mathrm{SiO}_{2}$ using plasma enhanced chemical vapor deposition (PECVD). The thickness of the dielectric layer is verified using a spectrographic method. Finally, the last 100 $\mathrm{nm} \mathrm{Al}$ layer is deposited on top of the $\mathrm{SiO}_{2}$. The thickness and conductivity of the top Al layer are verified using the above mentioned tools. Square pattern is photolithographically transfered and etched using the wet chemical etching. The optical micrograph of the fabricated structure is shown in Figure 4.
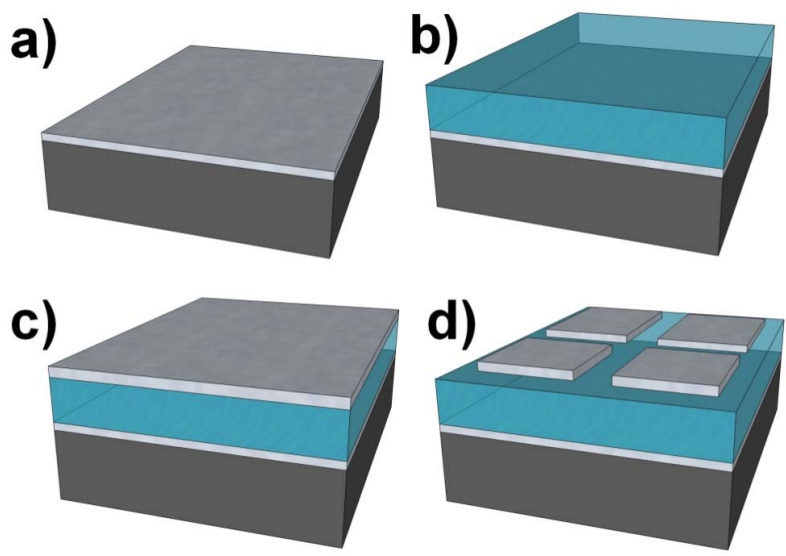

Figure 3. Microfabrication steps to produce the metamaterial a) $100 \mathrm{~nm}$ of $\mathrm{Al}$ is deposited on $\mathrm{Si}$ substrate using e-beam evaporation, b) $1.4 \mu \mathrm{m}$ of $\mathrm{SiO}_{2}$ is deposited using PECVD, c) another $100 \mathrm{~nm} \mathrm{Al}$ layer is deposited and d) top Al layer is photolithographically patterned and $16.5 \mu \mathrm{m} \times 16.5 \mu \mathrm{m}$ squares are wet-etched.

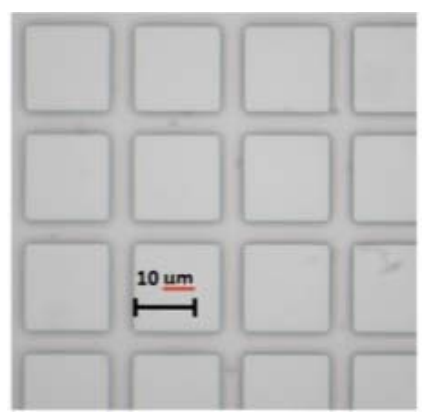

Figure 4. SEM micrograph of a section of the finished metamaterial structure on a Si wafer.

\section{EXPERIMENTAL MEASUREMENTS}

The optical characterization of the fabricated broad band and resonant absorbers were performed in the $3-5 \mathrm{THz}$ spectral band using a Fourier transform infrared spectrometer (FTIR Nexus 8700) fitted with globar source, Si beamsplitter and a pyroelectric detector.

In order to experimentally determine the absorption in the $\mathrm{THz}$ band, equation (1) was used, where the transmission and reflection coefficients were measured by placing the structure in the sample compartment of the FTIR as illustrated in Fig. 5 (a) and (b), respectively. From Fig. 5 b), it is apparent that the reflection measurement had to be taken at off-normal incidence (30 degrees in our setup). Thus, the transmission coefficient was experimentally measured by placing the sample at 30 degrees to the incident $\mathrm{THz}$ beam from the spectrometer. This configuration did not affect our resonant absorber calculations as we have modeled different angles and obtained very little change $(\sim 1 \%)$ between 0 to 30 degrees for our frequencies of interest. The background signal used for FTIR measurement for transmission was done without the sample in the path of the $\mathrm{THz}$ beam while for the background signal for the reflection measurement a gold-coated mirror was placed at the location of the sample in Fig. 5 a) to steer the beam from the source to the detector.

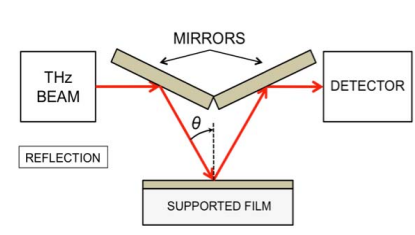

(a)

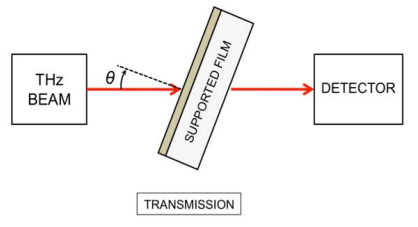

(b)
Figure 5 Schematics of a) reflection measurements where mirrors are used to stir the beam from the source to the sample and then to the detector (full background signal is obtained when the sample is replaced by another mirror and b) transmission measurements where the full signal is obtained by recording the unobstructed siganal from the source to the detector. Experimental measurements are performed at 30 degrees incident angle.

\section{RESULTS}

Figure 6 shows measured absorption as a function of $\mathrm{THz}$ frequency for a $10 \mathrm{~nm}$ thick $\mathrm{Ni}$ film on $\mathrm{Si}$ substrate. The periodic fringes observed in both simulation and in the experiment are caused by the Fabry-Perot effect. The $300 \mu \mathrm{m}$ $\mathrm{Si}$ wafer introduces an additional interface and the total reflected wave is a superposition of the portions of the wave reflected from all the interfaces, exhibiting constructive and destructive interference. The simulation of the absorption of the thin-film including the substrate shows good agreement with that of the measurements. Simulations of unsupported metal layer showed a flat absorption line (see Fig. 6) for in the case of thin-film absorber.

As mentioned above, the reflection measurement was the only tool needed to characterize the metamaterial absorbers. Plots in Figure 7 show a good agreement between the simulation and experimental measurement. The reflection of 
close to $0 \%$ at about $4.1 \mathrm{THz}$ corresponds to almost $100 \%$ absorption.

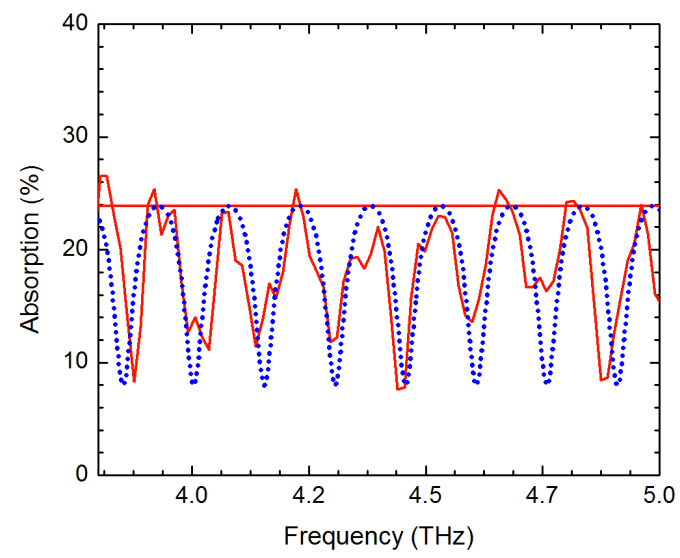

Figure 6 Plots in blue represent the finite element model predicted absorption while the measured values are shown in red, for a $10 \mathrm{~nm}$ Ni film depposited on a $300 \mu \mathrm{m}$ Si substrate. The straight line represents the absortpion of the unsupported film.

Further analysis shows that the absorption of the broadband absorbers can be controlled by the thin-film thickness (which, in turn, controls conductivity at nanometer scale) and that the resonant frequency of metamaterials can be tuned by varying the square dimensions and thickness of dielectric layers.

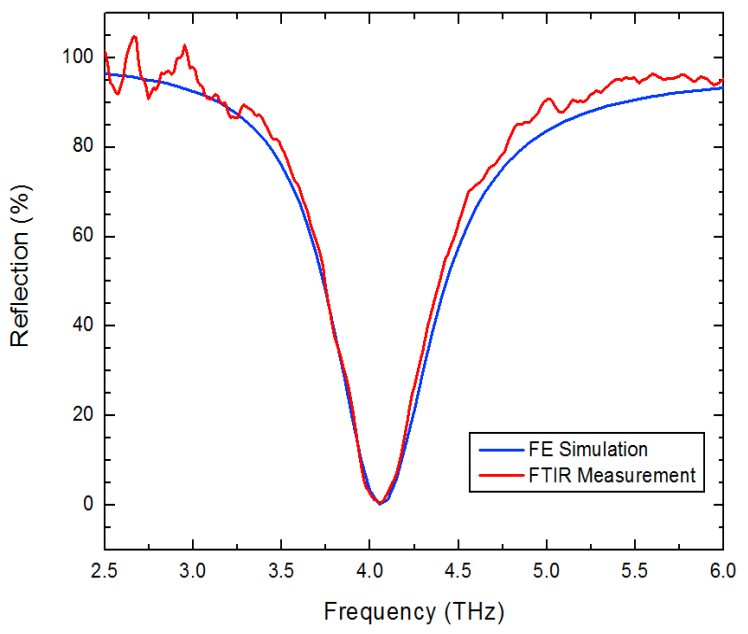

Figure 7 Plot of simulated and experimental reflection from the metamaterial wafer. The resonant reflection of almost 0 (corresponding to close to total absorption) occurs at $4.1 \mathrm{THz}$ for this sample.

\section{CONCLUSIONS}

By experimental verification, we demonstrated that it is possible to accurately model the absorption properties of both thin films and multi-layered metamaterials. This allows the design of both broad-band thermal sensors for spectral applications and highly-absorptive sensors for imaging applications where quantum cascade lasers are used as external illuminator. Both types of sensors can be designed to have optimal absorption properties in the desired frequency range.

\section{ACKNOWLEDGMENT}

This work is supported in part by a grant from the ONR. This research was in part conducted at the Center for Nanophase Materials Sciences, which is sponsored at Oak Ridge National Laboratory by the Office of Basic Energy Sciences, U.S. Department of Energy.

\section{REFERENCES}

[1] H. Tao, W. J. Padilla, X. Zhang, and R. Averitt, "Recent progress in electromagnetic metamaterial devices for Terahertz applications," IEEE J. Selec. Top. in Quantum Electronics, 17(1), pp (2011)

[2] B. S. Williams, H. Callebaut, S. Kumar, Q. Hu, and J. L. Reno "3.4$\mathrm{THz}$ quantum cascade laser based on longitudinal-optical-phonon scattering for depopulation" Appl. Phys. Lett. 82, 1015 (2003); doi:10.1063/1.1554479 (3 pages)

[3] B. N. Behnken, G. Karunasiri, D. R. Chamberlin, P. R. Robrish, and J Faist., "Real-time imaging using a $2.8 \mathrm{THz}$ quantum cascade laser and uncooled infrared microbolometer camera," Opt. Lett., 33(5), 440 (2008)

[4] D. Grbovic, "Imaging by Detection of Infrared Photons Using Arrays of Uncooled Micromechanical Detectors," in Department of Physics and Astronomy (University of Tennessee, Knoxville, TN, 2008).

[5] D. Grbovic, and G. Karunasiri, "Fabrication of Bi-material MEMS detector arrays for $\mathrm{THz}$ imaging," Proc. SPIE, (2009), p. 731108.Bolakis, Optics Express

[6] C. Bolakis, D. Grbovic, N. V. Lavrik, and G. Karunasiri, "Design and characterization of terahertz-absorbing nano-laminates of dielectric and metal thin films," Opt. Express 18, 14488-14495 (2010) 